\title{
Инструменты реализации кластерных программ в странах ОЭСР
}

\author{
Бондарева Я.Ю. \\ Белгородский государственный национальный исследовательский университет, \\ Россия, 308015, г. Белгород, ул. Победы, 85. \\ Bondareva_ya@bsu.edu.ru
}

\begin{abstract}
Аннотация. Активное развитие кластерной политики, кластеризация территорий во всем мире свидетельствуют о высокой эффективности кластеров, о возможности достижения высоких темпов экономического роста. В зарубежной практике кластеризация является основным элементом инновационной политики и служит для решения задач по освоению и комплексному развитию территорий. Сопоставляя лучший зарубежный опыт, авторы выявляют и обосновывают ключевые инструменты, используемые при кластеризации территорий. Несмотря на многочисленность публикаций по этой проблематике, практически отсутствуют исследования по системному анализу инструментов, используемых в кластерных программах зарубежных государств. Целью статьи является освещение различных инструментов, используемых в кластерных программах в странах членах ОЭСР. Рассматриваются категории часто используемых инструментов, в частности, для привлечения участников, предоставления коллективных услуг и содействия совместным исследованиям. Обсуждаются вопросы продолжительности программы и финансирования. Проводится анализ взаимодействия и взаимосвязей между программами для реализации самых разных типов кластеров. В результате исследования выявлены и проанализированы основные инструменты, позволяющие наиболее эффективно реализовать программы кластеризации территорий, поддерживающие региональную специализацию и развитие кластеров.
\end{abstract}

Ключевые слова: инструменты кластерных программ, кластерные связи, концепция кластера, региональная экономика, теоретические преимущества кластеризации, глобализация, сети.

Благодарности: исследование выполнено в рамках государственного задания НИУ «БелГУ» FZWG2020-0016 (0624-2020-0016), тема проекта «Фундаментальные основы глобальной территориальноотраслевой специализации в условиях цифровизации и конвергенции технологий».

Для цитирования: Бондарева Я.Ю. 2021. Инструменты реализации кластерных программ в странах ОЭСР. Экономика. Информатика, 48 (3): 426-433. DOI 10.52575/2687-0932-2021-48-3-426-433.

\section{Instruments for implementing cluster programs in OECD countries}

\author{
Yana Yu. Bondareva \\ Belgorod National Research University \\ 85 Pobeda St, Belgorod, 308015, Russia \\ Bondareva_ya@bsu.edu.ru
}

\begin{abstract}
The intensive development of industrial clusters around the world is due to broad opportunities for economic growth and positive multiplier effects. In international practice, the formation of a cluster strategy is considered as the most important component of innovation policy, demonstrating a balanced long-term approach of the state to the integrated development of territories. Comparing the best foreign experience, the authors identify and substantiate the key tools used in the clustering of territories. Despite the large number of publications on this topic, there are practically no studies on the system analysis of the tools used in the cluster programs of foreign countries. The purpose of the article is to highlight the various tools used in cluster programs in OECD countries. Categories of commonly used tools are considered, for attracting participants, providing collective services, and fostering collaborative research. The issues of program duration and funding
\end{abstract}


are discussed. An analysis of the interaction and relationships between programs for the implementation of various types of clusters is carried out. As a result of the study, the main tools have been identified and analyzed, which allow the most effective implementation of programs for clustering territories that support regional specialization and development of clusters.

Keywords: cluster software tools, cluster communications, cluster concept; regional economy; theoretical benefits of clustering; globalization; networks.

Acknowledgements: the study was carried out within the framework of the state assignment of NRU BelSU FZWG-2020-0016 (0624-2020-0016), the theme of the project is "Fundamental foundations of global territorial-sectoral specialization in the context of digitalization and convergence of technologies."

For citation: Bondareva Ya.Yu. 2021. Instruments for implementing cluster programs in OECD countries. Economics. Information technologies, 48 (3): 426-433 (in Russian). DOI 10.52575/2687-0932-2021-48-3426-433.

\section{Введение}

Инструменты реализации программ, поддерживающих региональную специализацию и развитие кластеров, направлены на использование теоретических преимуществ, включающих в себя основные сетевые преимущества масштаба, традиционные внешние эффекты Маршалла [Маршалл, 1993] объединение рынков труда, более высокий уровень специализации и, следовательно, доступ к услугам более высокого порядка, а также побочные эффекты знаний, Алмаз Портера [Porter, 2003], стимулирующий широкомасштабные инновации, а также более сложные инновационные процессы. В то время как преимущества приводят к повышению эффективности инноваций и специализации фирмы, для достижения этих преимуществ можно использовать различные инструменты. В этой статье мы обсудим несколько вопросов, связанных с:

1. Категорией инструментов. Большинство программ сосредоточены на одном или нескольких критериях инструментов для:

- привлечения участников;

- предоставления коллективных услуг;

- содействие совместным исследованиям.

2. Сроки осуществления программ и материальное обеспечение. Выделяют три типа финансирования:

- привлечение субъектов с бюджетами менее 100000 евро на кластер ежегодно и финансирование, как правило, в течение трех лет или менее;

- более существенные коллективные услуги и «легкие» инвестиции в НИОКР с ежегодными расходами на кластер от 100000 до 1 млн евро;

- «тяжелые» НИОКР, часто на длительный срок, до десяти лет. В некоторых случаях сроки реализации программы короче, чем можно было бы ожидать для успешного достижения заявленной цели [Amin, Cohendet, 1999].

В то время как некоторые программы действительно требуют совместного финансирования с разными уровнями правительства или частным сектором, так называемое государственно-частное партнерство, эффект привлечения частных средств, по-видимому, недостаточно развит во многих программах.

3. Создание синергетического эффекта за счет взаимосвязей. Важно отметить, что объединение несколькими странами инструментов в рамках различных программ по различным параметрам, таким как жизненный цикл продукта или стадия разработки кластерной инициативы, дает возможность предложить полный спектр инструментов поддержки кластеров. Существуют примеры объединения кластеров одних и тех же отраслей в разных географических точках или в разных отраслях, но по общей теме. Такие объединения позволяют достичь более эффективных результатов и служат примером пространственного взаимодействия кластерных структур. 
Таким образом, тезисы статьи актуальны для дальнейшего изучения, приращения различных точек зрения при обсуждении подобной темы. Практическая сторона исследования заключается в использовании данного материала как базиса при построении кластерной модели на региональном уровне. Методология исследования носит междисциплинарный характер и базируется преимущественно на методе системного анализа и сопоставления.

\section{Результаты и их обсуждение}

В общем виде инструменты, используемые программами в тематических исследованиях, бывают трех различных типов:

- для привлечения участников;

- для разработки коллективных услуг;

- для поддержки совместных исследований и разработок.

Общий обзор этих инструментов по категориям представлен на рисунке 1. Привлечение участников часто является необходимым условием для участия в коллективных услугах или в качестве компонента совместного проекта НИОКР. В ходе одного обзора кластеров были определены три важнейших фактора успеха для развития кластеров, на которых инструменты могли бы сосредоточиться: сети и партнерские отношения, сильная база навыков и потенциал в области инноваций и НИОКР [DTI, 2004]. Помимо этих общих факторов успеха, потребность в инструментах может варьироваться в зависимости от различных форм кластера, этапов жизненного цикла кластера и т. д. Бюджеты и временные рамки программ сильно различаются в зависимости от того, какие из этих типов инструментов используются.

Необходимо постоянно проводить анализ государственных стратегий для создания более благоприятной среды для кластеров.

Важным моментом является процесс привлечения участников программ. Так программы, в которых используются инструменты для привлечения участников, являются более эффективными. Создание сетей и партнерских отношений (т. е. взаимодействие между фирмами, между фирмами и другими субъектами) приносит ожидаемые результаты. Эти инициативы также могут быть сосредоточены либо на внутренних связях внутри кластера, либо на внешних связях между кластером и другими субъектами или регионами [Cortright, 2006]. Цель этих инструментов состоит не только в том, чтобы привлечь участников вместе, но для того, чтобы организовать их вокруг ключевых вопросов по отраслям или общей темы, охватывающей несколько отраслей [Competitive Regional Clusters - OECD, 2008].

Необходимо рассмотреть существующие на сегодняшний день формы кластерных инициатив. Так организации, которые управляют кластерными инициативами, принимают различные формы. К основным относятся: некоммерческие ассоциации; университет или аналогичные назначенные агенты; государственные учреждения. Они обычно принимают форму некоммерческой ассоциации, когда цель состоит в том, чтобы иметь отдельный правовой статус.

Еще одним важным элементом является уровень вовлеченности. Количество участников, участвующих в инициативе, является показателем их уровня ответственности и вовлеченности. Группы должны быть инклюзивными, однако по мере их расширения прямое участие субъектов может быть сокращено. В некоторых программах устанавливается минимальное число участников для получения финансирования [Competitive Regional Clusters OECD, 2008].

Большое значение приобретают совместные исследования и разработки.

Несмотря на высокую эффективность функционирования инновационных систем необходимо применять инструменты, позволяющие устранить имеющиеся слабые места. Многие инструменты поощрения инноваций предназначены для преодоления четко выявленных недостатков в национальных инновационных системах и их эффективности. Например, в НИОКР Франции слишком сильно доминирует государственный сектор, что 
приводит к отсутствию ориентации на рынок. Ожидается, что инициативы Германии в этой области будут направлены на устранение ощущаемого отсутствия эффективного сотрудничества между промышленностью и исследованиями (университетский сектор) и недостаточно скоординированной деятельности по поддержке исследований.

Привлечение участников
•Определение кластеров
(Проведение
картографических
исследований кластеров
(количественных и
качественных). Помощь
посредников и других
брокеров для выявления
фирм, потенциальных
партнеров.)
•Поддержка сетей, кластеров
(Проведение мероприятий
по повышению
осведомленности
(конференции, кластерное
образование). Применение
финансовых стимулов для
сетевых организаций, фирм.
Финансирование сетевых
мероприятий)

Поддержка совместных исследований и разработок

\section{-Усиление связи между} исследованиями и потребностями субъекта кластера (Поддержка совместных проектов между фирмами, университетами и научно-исследовательскими институтами. Совместное размещение различных участников для облегчения взаимодействия (например, научные парки, инкубаторы). Информационнопропагандистские программы университетов. Технические обсерватории.

\section{- Коммерциализация} исследований (Обеспечение соответствующих законов об интеллектуальной собственности. Преодоление барьеров для стимулирования государственного сектора в коммерциализации. Услуги по поддержке передачи технологий)

-Доступ к финансированию (Консультационные услуги по неординарным финансовым операциям. Программы государственных гарантий и венчурный капитал.

Рамочные условия поддержки частного венчурного капитала) Разработка коллективных
услуг

-Повышение потенциала, масштабов и квалификации поставщиков, главным образом МСп (Поддержка развития малого и среднего бизнеса. Посреднические услуги и платформы между поставщиками и покупателями. Сбор общей информации о рынке, координация закупок. Установление технических стандартов).

- Расширение внешних связей (Маркетинг кластеров и регионов. Помощь иностранным инвесторам в кластере. Рыночная информация для международных целей. Поиск партнеров. Поддержка связи в цепочке поставок, экспортные сети).

-Квалифицированная рабочая сила в стратегических отраслях

(Сбор и распространение информации о рынке труда. Специализированная профессиональная и университетская подготовка. Поддержка партнерских отношений между группами фирм и учебными заведениями.

Образовательные возможности)

Рис. 1. Инструменты региональной специализации и развития кластеров Fig. 1. Tools for regional specialization and cluster development

Недавние региональные инновационные инициативы в Италии являются частью более общей реакции на озабоченность политиков тем, что происходит отставание от других развитых европейских стран в отношении некоторых ключевых показателей эффективности в области НИОКР и инноваций. Например, расходы на НИОКР в бизнесе, уровень высшего и непрерывного образования, ЕС и международные патентование и другие показатели ниже, чем в среднем по ЕС. В Швеции озабоченность так называемым шведским парадоксом высоких расходов на НИОКР при низком уровне коммерциализации является ключевым фактором региональной инновационной и кластерной политики. В каждом случае акцент делался на создании синергетического эффекта.

Важным элементом является создание сетей и платформ. Учитывая первостепенность привлечения заинтересованных сторон в контексте совместных исследовательских проектов, 
большинство программ предусматривают соответствующие инструменты. Например, в шведской программe Vinnväxt по крайней мере 50 \% приемлемых расходов должны были быть потрачены на исследования и разработки, но другие приемлемые расходы включали управление процессами, создание бренда, организацию и стратегическую работу. В рамках Национальной кластерной программы Финляндии, которая в основном заключалась в совместных исследованиях и разработках, $25 \%$ средств было потрачено на управление кластерами. Во Франции для проведения конкурса требуются новые формальные структуры в качестве ключевого элемента программы. Тем не менее многие кластерные программы не всегда связаны с существующими исследовательскими платформами.

В некоторых случаях эти платформы и сети продвигаются через исследовательские парки, промышленные комплексы и другие транспортные средства. Достигнуты неоднозначные результаты в отношении эффективности и действенности таких инструментов для содействия расширению инноваций в контексте совместных исследований [Garofoli, 1984]. Иногда крупномасштабный проект действительно достигает успеха, например, Исследовательский треугольник Северной Каролины в Соединенных Штатах. Хотя необходимо отметить, что строительство с нуля - длительный и дорогостоящий процесс. Научно-промышленный парк Синьчжу в китайском Тайбэе начался в 1980 году с правительственного мандата и более чем 20 лет спустя - это кластер из почти 100000 сотрудников, двух университетов и 335 фирм и исследовательских центров [Lundmark, Power, 2003]. Во Франции София-Антиполис началась по инициативе правительства в регионе без промышленных или университетских традиций. Часто эти комплексы являются инструментами, спонсируемыми на региональном или местном уровнях, и поэтому необходимо четко увязать их с отдельной кластерной программой.

Далее необходимо рассмотреть вопросы коммерциализации. Программы включают ряд инструментов, помимо финансирования совместных научно-исследовательских проектов с фирмами для поддержки коммерциализации. В Японии, например, программа Кластера знаний включала в свою деятельность патентных юристов. Исследовательский альянс Грузии, среди прочего, предоставляет консультационные услуги исследователям. Рамочные условия также могут быть существенным препятствием для коммерциализации НИОКР, но эти вопросы решаются за пределами кластерных программ [Drabenstott M., 2005].

Большое значение приобретает содействие развитию предпринимательства и созданию фирм. На инструменты предпринимательства акцент делается только в некоторых программах с четкой инновационной ориентацией, несмотря на преимущества малых фирм в инновационных системах, учитывая их потенциал «созидательного разрушения». Так финский и норвежский Центры экспертизы активно связаны с программами научных парков и инкубаторов в своих странах. Фактически, финская программа даже включает в свою оценку успеха количество созданных новых компаний. Исследовательский альянс Грузии поддерживает проекты с университетскими партнерами, в том числе коммерциализацию исследований путем создания новых дочерних фирм, предоставляющих консультационные услуги и консультации по вопросам управления. В Японии Программа промышленного кластера имеет сильную направленность на создание МСП и направлена на создание возможностей для обучения предпринимателей. Корейские инновационные Города-кластеры часто включают компонент инкубатора. Инструменты для финансирования этих исследовательских дочерних компаний, такие как государственные фонды венчурного капитала, использовались лишь в нескольких из рассмотренных программ [Сарафанова, Павлова, Анастасова, 2018].

Анализируя опыт разных стран, можно выявить связь различных программ, инструментов и кластеров. Появляются так называемые взаимодополняемые программы. Поскольку ни одна политика или программа не может охватывать все инструменты, одним из решений является обеспечение того, чтобы различные программы эффективно дополняли друг друга [Cortright, 1994]. В Японии промышленный кластер и кластерные программы дополняют друг друга на протяжении всего производственного цикла. Кластеры знаний сосредоточены на поддержке 
университетских кластеров-хабов для передачи НИОКР. Программа промышленного кластера предназначена для поддержки существующих и вновь создаваемых МСП посредством создания сетей и коллективных услуг. Так, Япония стремится обеспечить успех этой взаимодополняемости через органы национального и регионального уровня с участием представителей обеих программ. В Швеции эта взаимодополняемость была также реализована в рамках программ Vinnväxt и Visanu, причем первые были в большей степени сосредоточены на проектах НИОКР, а вторые на общем развитии кластеров и деловых связях.

Появляются и дополнительные инструменты. Исследовательский альянс Грузии предлагает пакет инструментов, которые также служат различным этапам развития - от поиска исследователей до коммерциализации идей. Первым шагом является привлечение квалифицированных исследователей, а вместе с ними и талантливых и целеустремленных аспирантов. Программа «Выдающиеся ученые» служит для передачи экспертных знаний государству. GRA также спонсирует лаборатории и оборудование, которые предоставляются промышленным и университетским исследователям для поддержки исследований. Программа VentureLab предлагает услуги предварительного инкубатора, которые помогут университетам определить лабораторные открытия, которые обладают коммерческим потенциалом и которые помогут преподавателям пройти различные этапы разработки технологий до этапа создания компании. Награды Инновационного фонда GRA присуждаются преподавателям университетов, которые сотрудничают с фирмами в разработке и внедрении технологий.

Центры разработки технологий (технологические инкубаторы) помогают развивающимся компаниям получить доступ к ресурсам исследований и разработок принимающих университетов, одновременно совершенствуя коммерческий потенциал разрабатываемых технологий. В дополнение к специализированному оборудованию и помещениям, компании-инкубаторы имеют доступ к целому ряду услуг по запуску бизнеса и доступным помещениям, дополняют друг друга по кластерной стадии развития. Некоторые страны, регионы рассматривают свои программы как взаимодополняющие, причем одна из них служит в качестве предварительного отбора или конвейера для определения кластеров для другой программы [Drabenstott, 2005]. Так обстоит дело, например, в Норвегии, Орегоне (США) и Швеции. Программа «Арена» является гибкой и открытой для перспективных инициатив и предназначена для поддержки их развития.

Большинству программ требуется около года для начального этапа, прежде чем они будут профинансированы на основной проект, который обычно длится около двух лет. Программа Центров экспертизы предназначена для отбора уже функционирующих кластеров, которые стремятся повысить уровень сотрудничества в области НИОКР и интернационализироваться. Конкурсный отбор и долгосрочное финансирование (десятилетние циклы) являются условиями для программы, на которую могут претендовать лучшие сети. Кластерная сеть штата Орегон стремится поддерживать все кластеры, заинтересованные в развитии. OregonInC, отдельная организация, разработает программы для обслуживайте тех, кто был признан успешным. В Швеции программа Visanu была нацелена на многие инициативы, которые не получили финансирования.

Учитывая, что потенциальным недостатком конвейерного подхода является исключение новых перспективных кластеров, следует рассмотреть возможность сохранения программы, открытой для кандидатов, которые не были в процессе разработки. В ходе последовательных этапов финансирования одной и той же программы цели могут дополнять друг друга. Например, Vinnväxt основал в первых двух этапах наиболее перспективные кластеры, проекты. Третий этап сосредоточен на большем количестве эмбриональных кластеров. Как японские, так и корейские программы рассматривают свои программы в более долгосрочной перспективе с отдельными этапами. Например, Национальный план сбалансированного развития рассматривает инновационные программы в три этапа продолжительностью пять лет:

1) создание инновационных систем;

2) переход в инновационный кластер мирового класса;

3) укрепление региональной инновационной системы. 
Оценка промышленных кластеров Японии также предложила три пятилетних этапа для программы:

1) начальный период;

2) период роста;

3) период самообеспечения [Competitive Regional Clusters - OECD, 2008].

Эти различные этапы предполагают необходимость в дополнительных инструментах с течением времени.

Важным моментом является обмен информацией.

Помимо обмена базовой информацией, кластерные связи могут быть как межсекторальными связями для развития кластера, так и связями между кластерами в одних и тех же областях. Межсекторальные связи служат развитию новых тематических блоков [Competitive Regional Clusters - OECD, 2008].

Такие примеры кластерных связей позволяют судить о высоком уровне развития кластеризации территорий в западных странах и могут служить примером для формирования кластерных связей в РФ.

\section{Заключение}

В статье были рассмотрены инструменты реализации кластерной политики, применяемые в странах членах ОЭСР, поддерживающие региональную специализацию и кластеризацию территорий, направленные на использование теоретических преимуществ, в частности категории инструментов, продолжительности программ и особенностей их финансирования, создание синергетического эффекта за счет взаимосвязей. Так же были изучены и проанализированы основные примеры реализации инструментов, представленных и действующих в зарубежных странах в рамках НИОКР, рассмотрены способы обмена информацией, описаны кластерные связи и возможные методы привлечения дополнительных инструментов в межсекторальных связях.

Таким образом, было выявлено, что инструменты, используемые в кластерных программах разных стран, позволяют наиболее эффективно решать задачи директивных органов на национальном уровне по кластеризации территорий, учитывающих широкий спектр типов кластеров или ориентированных на те кластеры, которые могут помочь в достижении определенных целей. Инструменты кластерной политики могут применяться в развитых и отстающих регионах, для систем малого и среднего бизнеса или крупных фирм, для обслуживания новых и зрелых отраслей, для ориентации на существующие отрасли или для создания новых отраслей.

\section{Список источников}

1. Competitive Regional Clusters - OECD. 2008. URL: http://www.docme.ru/doc/1204601/ 283.competitive-regional-clusters-oecd (дата обращения: 05.07.2021).

2. DTI. 2004. Benchmarking UK productivity performance: The Government's response to the consultation on productivity indicators, $12 \mathrm{p}$.

\section{Список литературы}

1. Маршалл А. 1993. Принципы экономической науки. М., Издательская группа «Прогресс», 23 с.

2. Сарафанова А.Н., Павлова А.С., Анастасова А.С. 2018. Институциональные формы развития территорий: технопарки и кластеры. OPEN INNOVATION: сб. ст. III Междунар. науч.-практ. конф. Пенза: МЦНС «Наука и просвещение»: 89-92.

3. Amin A., Cohendet P. 1999. Learning and Adaptation in Decentralised Business Networks, Environment and Planning D: Society and Space, 17: 87-104.

4. Andersson T., Napier G. 2004. The Venture Capital Market - Global Trends and Issues for Nordic Countries, Malmö.

5. Cortright J. 1994. Reinventing Economic Development: Ten Ideas for Market Driven Approaches to Promoting Industrial Competitiveness, Staff Report to the Joint Legislative Committee on Trade and Economic Development, October, 33 p. 
6. Cortright J. 2006. Making sense of clusters: regional competitiveness and economic development. Washington, D.C.: Brookings Institution, Metropolitan Policy Program.

7. Drabenstott M. 2005. A review of the federal role in regional economic development, Report for the Center for the Study of Rural America, Federal Reserve Bank of Kansas City, May.

8. Garofoli G. 1984. Barriere all'innovazione e politiche d'intervento a livello regionale e subregionale, in Camagni, R., Cappellin, R. and Garofoli, G. (eds.), Cambiamento tecnologico e diffusione territoriale. Scenari regionali di risposta alla crisi, Franco Angeli, Milano.

9. Lall S. 1980. Developing Countries as Exporters of Technology and Capital Goods; the Indian Experience, mimeo, Oxford University Institute of Economics and Statistics, Oxford.

10. Lundmark M., Power D. 2003. Working through Knowledge Pools: Labour Market Dynamics, the Transference of Knowledge and Ideas, and Industrial Clusters. Urban Studies, 41: 5-6.

11. Pavitt K. 1998. Technologies, Products and Organization in the Innovating Firm: What Adam Smith Tells Us and Joseph Schumpeter Doesn't. Industrial and Corporate Change, 7(3): 433-452.

12. Porter M.E. 2003. The economic performance of regions. Regional Studies, 37(6-7):545-546.

\section{References}

1. Marshall A. 1993. Principy ekonomicheskoj nauki [Principles of Economic Science]. M., Publishing group "Progress", 23 p.

2. Sarafanova A.N., Pavlova A.S., Anastasova A.S. 2018. Institucional'nye formy razvitiya territorij: tekhnoparki i klastery [Institutional forms of development of territories: technology parks and clusters]. OPEN INNOVATION: collection of works. Art. III Int. scientific-practical conf. Penza: ICNS "Science and Education": 89-92.

3. Amin A., Cohendet P. 1999. Learning and Adaptation in Decentralised Business Networks, Environment and Planning D: Society and Space, 17: 87-104.

4. Andersson T., Napier G. 2004. The Venture Capital Market - Global Trends and Issues for Nordic Countries, Malmö.

5. Cortright J. 1994. Reinventing Economic Development: Ten Ideas for Market Driven Approaches to Promoting Industrial Competitiveness, Staff Report to the Joint Legislative Committee on Trade and Economic Development, October, 33 p.

6. Cortright J. 2006. Making sense of clusters: regional competitiveness and economic development. Washington, D.C.: Brookings Institution, Metropolitan Policy Program.

7. Drabenstott M. 2005. A review of the federal role in regional economic development, Report for the Center for the Study of Rural America, Federal Reserve Bank of Kansas City, May.

8. Garofoli G. 1984. Barriere all'innovazione e politiche d'intervento a livello regionale e subregionale, in Camagni, R., Cappellin, R. and Garofoli, G. (eds.), Cambiamento tecnologico e diffusione territoriale. Scenari regionali di risposta alla crisi, Franco Angeli, Milano.

9. Lall S. 1980. Developing Countries as Exporters of Technology and Capital Goods; the Indian Experience, mimeo, Oxford University Institute of Economics and Statistics, Oxford.

10. Lundmark M., Power D. 2003. Working through Knowledge Pools: Labour Market Dynamics, the Transference of Knowledge and Ideas, and Industrial Clusters. Urban Studies, 41: 5-6.

11. Pavitt K. 1998. Technologies, Products and Organization in the Innovating Firm: What Adam Smith Tells Us and Joseph Schumpeter Doesn't. Industrial and Corporate Change, 7(3): 433-452.

12. Porter M.E. 2003. The economic performance of regions. Regional Studies, 37(6-7):545-546.

Конфликт интересов: о потенциальном конфликте интересов не сообщалось.

Conflict of interest: no potential conflict of interest related to this article was reported.

\section{ИНФОРМАЦИЯ ОБ АВТОРЕ}

\footnotetext{
Бондарева Яна Юрьевна, кандидат экономических наук, доцент, доцент кафедры прикладной экономики и экономической безопасности Белгородского государственного национального исследовательского университета, г. Белгород, Россия
}

\section{INFORMATION ABOUT THE AUTHOR}

Yana Yu. Bondareva, Candidate of Economic Sciences, Associate Professor, Associate Professor of the Department of Applied Economics and Economic Security, Belgorod National Research University, Belgorod, Russia 